Discrete Comput Geom 32:383-400 (2004)

DOI: $10.1007 / \mathrm{s} 00454-004-1096-4$

\title{
Approximation of the Normal Vector Field and the Area of a Smooth Surface*
}

\author{
Jean-Marie Morvan ${ }^{1,2}$ and Boris Thibert ${ }^{2}$ \\ ${ }^{1}$ INRIA, 2004 Route des Lucioles, B.P 93, \\ 06904 Sophia-Antipolis, France \\ Jean-Marie.Morvan@sophia.inria.fr \\ ${ }^{2}$ Institut Girard Desargues, Université Claude Bernard Lyon1, \\ bât. 21, 43 Bd du 11 novembre 1918, \\ 69622 Villeurbanne Cedex, France \\ thibert@igd.univ-lyon1.fr
}

\begin{abstract}
This paper deals with the comparison of the normal vector field of a smooth surface $S$ with the normal vector field of another surface differentiable almost everywhere. The main result gives an upper bound on angles between the normals of $S$ and the normals of a triangulation $T$ close to $S$. This upper bound is expressed in terms of the geometry of $T$, the curvature of $S$ and the Hausdorff distance between both surfaces. This kind of result is really useful: in particular, results of the approximation of the normal vector field of a smooth surface $S$ can induce results of the approximation of the area; indeed, in a very general case ( $T$ is only supposed to be locally the graph of a lipschitz function), if we know the angle between the normals of both surfaces, then we can explicitly express the area of $S$ in terms of geometrical invariants of $T$, the curvature of $S$ and of the Hausdorff distance between both surfaces. We also apply our results in surface reconstruction: we obtain convergence results when $T$ is the restricted Delaunay triangulation of an $\varepsilon$-sample of $S$; using Chew's algorithm, we also build sequences of triangulations inscribed in $S$ whose curvature measures tend to the curvatures measures of $S$.
\end{abstract}

\section{Introduction}

In this paper we are interested in the relationship between the geometrical properties of a smooth surface $S$ and the geometrical properties of another surface "close" to $S$.

* This work was partially supported by the IST Programme of the EU as a Shared-cost RTD (FET Open) Project under Contract No. IST-2000-26473 (ECG - Effective Computational Geometry for Curves and Surfaces), and by Région Rhônes Alpes, Contrat 99029744. 
We wonder whether the geometrical properties of $S$ can be well approximated by the "approximating" surface. The normal vector field is an important property: indeed, we show that if the angle between the normal vector field of a smooth surface and the normal vector field of a locally lipschitz surface (i.e. a surface that is locally the graph of a lipschitz function) $M$ is known, then we can explicitly express the area of $S$ in terms of geometrical invariants of $M$, the curvature of $S$ and the Hausdorff distance between both surfaces.

Our main result gives an upper bound on the maximal angle $\alpha_{\max }$ between the normals of a smooth surface $S$ and the normals of a triangulation $T$ close to it that depends on the geometry of $T$, the curvature of $S$ and the Hausdorff distance between both surfaces. It is worth noting that this upper bound can be small even if some triangle's angles are very small. As corollaries, we bound $\alpha_{\max }$ from above with the largest circumradius of the triangles of $T$ (up to a constant that only depends on $S$ ) and we get the convergence's results.

These results are very general and must be compared with [14]. In the context of surface reconstruction from scattered sample points, Amenta et al. study the particular case of $\varepsilon$-samples (see [2] and [3]) and get a similar approximation: they build a triangulation (based on an $\varepsilon$-sample of $S$ ) whose triangle's circumradii are small and they deduce an approximation of the normals of $S$. In [10] Fu has results of convergence of the curvature measures of a sequence of triangulations to the curvature measure of a smooth surface. Our results only deal with the normal vector field and with the area, but they are quantitative and more precise: we compare explicitly two surfaces and we get the convergence's results as corollaries.

We give applications of our results in the theory of surface reconstruction from scattered sample points: in particular, if $\mathcal{S}$ is an $\varepsilon$-sample of a (closed) surface $S$ (in the sense of [1]), then the limit of the area $\mathcal{A}\left(T_{\varepsilon}\right)$ of the Delaunay triangulation $T_{\varepsilon}$ restricted to the $\varepsilon$-sample $\mathcal{S}$ satisfies

$$
\lim _{\varepsilon \rightarrow 0} \mathcal{A}\left(T_{\varepsilon}\right)=\mathcal{A}(S) .
$$

This paper is organised as follows. Section 3 states the results of the approximation of the normals of a smooth surface with the normals of a triangulation close to it. In Section 4 we first give the results of the approximation of the area of a smooth surface with the area of a locally lipschitz surface. As a corollary (and by using the results of Section 3) we deduce the results of the approximation when the approximating surface is a triangulation. Section 5 deals with applications in surface reconstruction: we prove a result of convergence concerning restricted Delaunay triangulations; using Chew's algorithm, we produce a sequence of triangulations which are close to $S$, and whose area tends to the area of $S$. Sections 6 and 7 sketch the proofs.

The background on differential geometry can be found in [4], [8], [12], and [15].

\section{Definitions}

We recall here some classical definitions which concern smooth surfaces, triangulations and the relative position of two surfaces. For more details on triangulations, one may refer to [10] and [6]. 


\subsection{Smooth Surfaces}

In the following, a smooth surface means a $\mathcal{C}^{2}$ surface which is regular, oriented, with or without boundary. Let $S$ be a smooth surface of the (oriented) Euclidean space $E^{3}$. Let $\partial S$ denote the boundary of $S$. $S$ is endowed with the Riemannian structure induced by the standard scalar product of $E^{3}$. We denote by $d a$ the area form of $S$ and by $d s$ the canonical orientation of $\partial S$. Let $v$ be the unitary normal vector field (compatible with the orientation of $S$ ) and let $h$ be the second fundamental form of $S$ associated with $v$. Its determinant at a point $p$ of $S$ is the Gauss curvature $G(p)$, its trace is the mean curvature $H(p)$. The maximal curvature of $S$ at $p$ is $\rho_{p}=\max \left(\left|\lambda^{1}(p)\right|,\left|\lambda^{2}(p)\right|\right)$, where $\lambda_{1}(p)$ and $\lambda_{2}(p)$ are the eigenvalues of the second fundamental form at $p$. Finally, the maximal curvature of $S$ is

$$
\rho_{S}=\sup _{p \in S} \rho_{p}
$$

We need the following:

Proposition 1. Let $S$ be a smooth compact (oriented) surface of $E^{3}$. Then there exists an open set $U_{S}$ of $E^{3}$ containing $S$ and a continuous map $\xi$ from $U_{S}$ onto $S$ satisfying the following property: if p belongs to $U_{S}$, then there exists a unique point $\xi(p)$ realising the distance from $p$ to $S$ ( $\xi$ is nothing but the orthogonal projection onto $S$ ).

A proof of this proposition can be found in [9].

The open set $U_{S}$ depends locally and globally on the smooth surface $S$. Locally, the reach is less than the radius of curvature. Globally, $U_{S}$ depends on points which can be far from one another on the surface, but close in $E^{3}$.

We shall also need the notion of the reach of a surface, introduced in [9].

Definition 1. The reach of a surface $S$ is the largest $r>0$ for which $\xi$ is defined on the (open) tubular neighbourhood $U_{r}(S)$ of radius $r$ of $S$.

This implies that the reach $r_{S}$ of $S$ is smaller than the minimal radius of curvature of $S$. Thus, we have

$$
\rho_{S} r_{S} \leq 1
$$

\subsection{Triangulations}

A triangulation $T$ is a topological surface which is a (finite and connected) union of triangles of $E^{3}$, such that the intersection of two triangles is either empty, or equal to a vertex, or equal to an edge.

We denote by $\mathcal{T}_{T}$ the set of triangles of $T$ and by $\Delta$ a generic triangle of $T$.

- $\eta(\Delta)$ denotes the length of the longest edge of $\Delta$, and $\mathcal{A}(\Delta)$ its area.

- The fatness of $\Delta$ is the real number

$$
\theta(\Delta)=\frac{\mathcal{A}(\Delta)}{\eta(\Delta)^{2}}
$$




\section{$\operatorname{rig}(\Delta)=1$}

Fig. 1. Rightness of a triangle $\Delta$.

- We introduce the notion of rightness of a triangle $\Delta$ (Fig. 1). It is the real number

$$
\operatorname{rig}(\Delta)=\sup _{p \text { vertex of } \Delta}\left|\sin \left(\theta_{p}\right)\right|,
$$

where $\theta_{p}$ is the angle at $p$ of $\Delta$.

Globally:

- The area $\mathcal{A}(T)$ is the sum of the areas of all the triangles of $T$.

- The height of $T$ is

$$
\eta(T)=\sup _{\Delta \in \mathcal{T}_{T}} \eta(\Delta)
$$

- The fatness of $T$ is

$$
\theta(T)=\min _{\Delta \in \mathcal{T}_{T}} \theta(\Delta)
$$

- The rightness of $T$ is

$$
\operatorname{rig}(T)=\min _{\Delta \in \mathcal{T}_{T}} \operatorname{rig}(\Delta) .
$$

Note that the rightness is a weaker condition than the fatness. The rightness of a triangle is large if there exists at least one angle whose sinus is large. The fatness of a triangle is large if the sinus of every angle is large.

\subsection{Surface Closely Near a Smooth Surface}

We introduce the following definitions:

- A subset $M$ of $E^{3}$ is closely near a smooth surface $S$ if it lies in $U_{r}(S)$, where $r$ is the reach of $S$ and if the restriction of $\xi$ to $M$ is one-to-one.

- Let $M$ be a subset closely near a smooth surface $S$. The relative curvature of $M$ with respect to $S$ is the real number defined by

$$
\omega_{S}(M)=\sup _{m \in M \backslash \partial M}\|\xi(m)-m\| \rho_{\xi(m)} .
$$

- If $M$ is a surface closely near $S$ and differentiable almost everywhere, one can define at almost every point $m$ of $M$ the angle $\alpha_{m} \in[0, \pi / 2]$ between the tangent planes $T_{m} M$ and $T_{\xi(m)} S$. We put

$$
\begin{aligned}
\alpha_{\max } & =\max _{m \in M} \alpha_{m}, \\
\alpha_{\min } & =\min _{m \in M} \alpha_{m} .
\end{aligned}
$$

$\alpha_{\max }$ is called the maximal angle between the normals of $S$ and $M$. 
Remark. If $M$ lies in $U_{r}(S)$, then $\omega_{S}(M) \leq 1$ (this is due to the fact that for every $m \in M$, the point $m-2 \overrightarrow{m \xi(m)}$ - which is the orthogonal symmetric of $m$ with respect to the tangent plane of $S$ at $\xi(m)$ - belongs to $\left.U_{r}(S)\right)$.

- We say that a triangulation of $E^{3}$ is inscribed in a smooth surface $S$ if all its vertices belong to $S$.

- A triangulation $T$ is closely inscribed in a smooth surface $S$ if:

1. $T$ is closely near $S$,

2. all vertices of $T$ belong to $S$,

3. all vertices of $\partial T$ belong to $\partial S$.

- The relative height of $T$ with respect to $S$ is the real number defined by

$$
\pi_{S}(T)=\sup _{\Delta \in \mathcal{T}} \sup _{m \in \Delta} \eta(\Delta) \rho_{\xi(m)} .
$$

Remark. Let $T$ be a triangulation closely inscribed in a smooth surface $S$. We have

$$
\omega_{S}(T) \leq \pi_{S}(T) .
$$

\section{An Approximation on the Normal Vector Field}

In this section we compare the behaviour of the normal vector field of a smooth surface $S$ with the normal of each face of a triangulation which is closely inscribed in $S$. We express the approximation in terms of the geometric invariants that we introduced in the previous section.

Theorem 1. Let $S$ be a smooth surface and let $T$ be a triangulation closely inscribed in $S$. Then the maximal angle $\alpha_{\max }$ between the normals of $S$ and $T$ satisfies

$$
\sin \alpha_{\max } \leq\left(\frac{\sqrt{10}}{2 \operatorname{rig}(T)\left(1-\omega_{S}(T)\right)}+\frac{1}{1-\omega_{S}(T)}\right) \pi_{S}(T) .
$$

Corollary 1. Let $S$ be a smooth surface and let $T$ be a triangulation closely inscribed in $S$. If

$$
\pi_{S}(T) \leq \frac{1}{2}
$$

then the maximal angle $\alpha_{\max }$ between the normals of $S$ and $T$ satisfies

$$
\sin \alpha_{\max } \leq\left(\frac{4}{\operatorname{rig}(T)}+2\right) \pi_{S}(T) .
$$

Corollary 2. Let $S$ be a (compact orientable) $C^{2}$ surface in $E^{3}$. Let $T_{n}$ be a sequence of triangulations closely inscribed in $S$. If

1. the length of the edges of $T_{n}$ tends to zero when $n$ goes to infinity,

2. the rightness of $T_{n}$ is (uniformly) bounded from below by a positive constant,

then the normals of $T_{n}$ tend to the normals of $S$. 
Since $S$ is compact, the second condition may be weakened by asking that $\pi_{S}\left(T_{n}\right)$ tends to zero when $n$ goes to infinity (in some sense, the length of the edges may be "large" when the curvature is "small"...).

Remark 1. The geometrical quantities $\pi_{S}(T)$ and $\operatorname{rig}(T)$ are linked to the circumradii of the triangles of $T$. If $\Delta$ is a triangle of $T$, and if $R_{\Delta}$ denotes the circumradius of $\Delta$, then we have

$$
\rho_{\xi(\Delta)} R_{\Delta}=\frac{\rho_{\xi(\Delta)} \eta_{\Delta}}{2 \operatorname{rig}(\Delta)}=\frac{\pi_{\Delta}(\xi(\Delta))}{2 \operatorname{rig}(\Delta)} .
$$

This implies the following:

Corollary 3. Let $S$ be a smooth surface and let $T$ be a triangulation closely inscribed in $S$. We get

$$
\alpha_{\max } \leq K(S)\left(\sup _{\Delta \text { triangle of } T} R_{\Delta}\right),
$$

where $K(S)$ is a constant depending on $S$ and $R_{\Delta}$ is the circumradius of $\Delta$.

\section{Approximation of the Area}

This section deals with the approximation of the area of a smooth surface $S$ with the area of a surface $M$ close to it.

1. In Section 4.1 we show that if a surface $M$ differentiable almost everywhere is close enough to a smooth surface $S$, and if the normals of $M$ are close to the normals of $S$, then the area of $M$ is close to the area of $S$. In other words, this means that the approximation of the normals induces results of the approximation of the area.

2. In Section 4.2 we deduce from Section 4.1 and from Section 3 results of the approximation of the area when $M$ is a triangulation.

\subsection{Locally Lipschitz Surfaces}

The following result shows that if $M$ is closely near $S$ and has enough regularity to have a tangent plane almost everywhere, then the area of $S$ is bounded from above and from below by quantities depending on the area of $M$, the Hausdorff distance between $M$ and $S$, the curvature of $S$ and the angle between the corresponding tangent planes.

A surface is said to be locally lipschitz if it is locally the graph of a lipschitz function. The chosen framework is the class of locally lipschitz surfaces (these surfaces are differentiable almost everywhere [13]; this class contains all the triangulations). 
Theorem 2. Let $S$ be a (compact orientable) $C^{2}$ surface in $E^{3}$. Let $M$ be a locally lipschitz surface closely near $S$. Then the area satisfies

$$
\mathcal{A}(S)=\int_{M} \frac{\cos \alpha_{m}}{1+\delta_{m} \varepsilon_{m} H_{\xi(m)}+\delta_{m}^{2} G_{\xi(m)}} d a_{M}(m),
$$

where $\delta_{m}=\|\xi(m)-m\|$ and $\varepsilon_{m} \in\{-1,+1\}$ is the scalar product between $\nu_{\xi(m)}$ and $(\xi(m)-m) /\|\xi(m)-m\|$.

The Hausdorff distance $\delta_{\text {Hauss }}$ between two subsets $A$ and $B$ of $E^{3}$ is defined by

$$
\delta_{\text {Hauss }}(A, B)=\max \left(\sup _{x \in A} d(x, B), \sup _{y \in B} d(A, y)\right) .
$$

Corollary 4. Let $M$ be a locally lipschitz surface closely near $S$.

1. Then

$$
\frac{\cos \alpha_{\max }}{\left(1+\omega_{S}(M)\right)^{2}} \mathcal{A}(M) \leq \mathcal{A}(S) \leq \frac{\cos \alpha_{\min }}{\left(1-\omega_{S}(M)\right)^{2}} \mathcal{A}(M) .
$$

2. In particular,

$$
|\mathcal{A}(S)-\mathcal{A}(M)| \leq K(S)\left(\alpha_{\max }^{2}+\omega_{S}(M)\right)
$$

and

$$
|\mathcal{A}(S)-\mathcal{A}(M)| \leq K(S)\left(\alpha_{\max }^{2}+\delta_{\text {Hauss }}(S, M)\right),
$$

where $K(S)$ is a constant depending on $S$.

From the previous corollary we deduce the following:

Corollary 5. Let $S$ be a (compact orientable) $C^{2}$ surface in $E^{3}$. Let $M_{n}$ be a sequence of locally lipschitz surfaces closely near S. If

1. the Hausdorff limit of $M_{n}$ is $S$, when $n$ goes to infinity,

2. the angle between tangent planes $T_{m} M_{n}$ and $T_{\xi(m)} S$ tends to zero almost everywhere, when $n$ goes to infinity,

then

$$
\lim _{n \rightarrow \infty} \mathcal{A}\left(M_{n}\right)=\mathcal{A}(S) .
$$

We now present two examples showing the relevance of the introduced geometric quantities:

1. First, consider two spheres $S$ and $M$ with same centres but different radii, 1 and $R$ (Fig. 2). The angle between the normals of $S$ and $M$ is 0 , but the relative curvature of $M$ with respect to $S$ is equal to $1-R$. If $R$ tends to 0 , then $\omega_{S}(M)$ tends to 1 and the area of $M$ to 0 .

2. Consider now a flat surface $S$, and a sequence $\left(M_{n}\right)_{n \geq 1}$ of locally lipschitz surfaces such that $\omega_{S}\left(M_{n}\right)$ is equal to 0 and $\alpha_{\max }$ is equal to $\pi / 4$ (Fig. 3). The area of $M_{n}$ is equal to $\sqrt{2}$ times the area of $S$. 


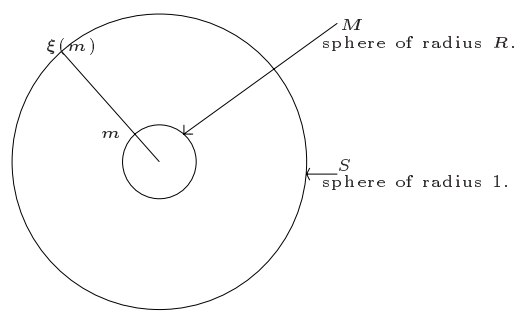

Fig. 2. $\operatorname{Cos} \alpha_{\max }=1$ and $\omega_{S}(M)$ is large.

\subsection{Approximations with Triangulations}

In Section 4.1 we got an approximation of the area of a smooth surface by the area of an almost-differentiable surface close to it, in terms of the angles between the corresponding normals. Using this result and the approximation of the normals (Corollary 1), we get the following:

Corollary 6. Let $S$ be a (compact orientable) $C^{2}$-surface in $E^{3}$ and let $T$ be a triangulation closely inscribed in $S$. If

$$
\left(\frac{4}{\operatorname{rig}(T)}+2\right) \pi_{S}(T) \leq 1,
$$

then the area of $S$ satisfies

$$
\frac{\sqrt{1-(4 / \operatorname{rig}(T)+2)^{2} \pi_{S}(T)^{2}}}{\left(1+\omega_{S}(T)\right)^{2}} \mathcal{A}(T) \leq \mathcal{A}(S) \leq \frac{1}{\left(1-\omega_{S}(T)\right)^{2}} \mathcal{A}(T) .
$$

As an obvious consequence, we get the following convergence result:

Corollary 7. Let $S$ be a (compact orientable) $C^{2}$ surface in $E^{3}$. Let $T_{n}$ be a sequence of triangulations closely inscribed in $S$. If

1. the length of the edges of $T_{n}$ tends to zero when $n$ goes to infinity,

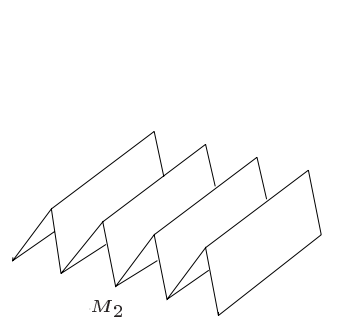

(a)
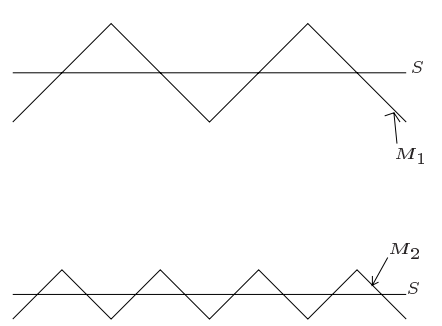

(b)

Fig. 3. $\operatorname{Cos} \alpha_{m}=\sqrt{2} / 2$ and $\omega_{S}\left(M_{n}\right)=0$. 
2. the rightness of $T_{n}$ is (uniformly) bounded from below by a positive constant, then

$$
\lim _{n \rightarrow \infty} \mathcal{A}\left(T_{n}\right)=\mathcal{A}(S)
$$

Using Remark 1, we also have:

Corollary 8. Let $S$ be a smooth surface and let $T$ be a triangulation closely inscribed in $S$. Then

$$
|\mathcal{A}(T)-\mathcal{A}(S)| \leq K(S)\left(\sup _{\Delta \text { triangle of } T} R_{\Delta}\right),
$$

where $K(S)$ is a constant depending on $S$ and $R_{\Delta}$ is the circumradius of $\Delta$.

\section{Applications in Surface Reconstruction}

Our results are general. However, they can be applied to particular algorithms of surface reconstruction.

\subsection{Restricted Delaunay Triangulations}

Let $\mathcal{S}$ be a finite set of points inscribed in a (compact without boundary) surface $S$. We recall the following [1]:

Definition 2. The set $T$ of Delaunay simplices of $\mathcal{S}$ whose dual intersects $S$ is called the restricted Delaunay triangulation of $\mathcal{S}$ with respect to $S$.

If the sample has suitable properties, then $T$ has interesting topological and geometrical properties. We use the concept of an $\varepsilon$-sample, introduced in [1]:

Definition 3. A set of points $\mathcal{S}$ on $S$ is an $\varepsilon$-sample of $S$ if and only if, for every point $m$ of $S$, the ball $B(m, \varepsilon$ lfs $(m))$ encloses at least one point of $\mathcal{S}$.

Let us summarise the main properties of the restricted Delaunay triangulation associated to an $\varepsilon$-sample. We denote by $T_{\mathcal{S}_{\varepsilon}}$ the restricted Delaunay triangulation of an $\varepsilon$-sample $\mathcal{S}_{\varepsilon}$ with respect to $S$. Amenta et al. [3] showed that if $\varepsilon<0.08$, then $T_{\mathcal{S}_{\varepsilon}}$ is closely inscribed in $S$. Furthermore, Amenta and Bern proved the following property [1]:

Proposition 2. The maximal angle $\alpha_{\max }$ between the corresponding tangent planes of $T_{\mathcal{S}_{\varepsilon}}$ and $S$ (via $\xi$ ) satisfies

$$
\alpha_{\max }=O(\varepsilon) .
$$


As an immediate consequence of Proposition 2 and Theorem 2, we get the following:

Corollary 9. Let $S$ be a compact without boundary surface of $E^{3}$. Let $T_{\mathcal{S}_{\varepsilon}}$ be the restricted Delaunay triangulation of an $\varepsilon$-sample $\mathcal{S}_{\varepsilon}$ with respect to $S$. Then

$$
\lim _{\varepsilon \rightarrow 0} \mathcal{A}\left(T_{\mathcal{S}_{\varepsilon}}\right)=\mathcal{A}(S) .
$$

\subsection{An Application Using Chew's Algorithm}

Let $S$ be a compact surface, let $\mathcal{S}$ be a $\tilde{\varepsilon}$-sample of $S(\tilde{\varepsilon}<0.1)$ and let $\operatorname{Del}_{S}(\mathcal{S})$ be the restricted Delaunay triangulation associated to the sample $\mathcal{S}$ of $S$. From $\operatorname{Del}_{S}(\mathcal{S})$, Chew's algorithm builds a triangulation which is finer than $\operatorname{Del}_{S}(\mathcal{S})$, and whose fatness is $>30^{\circ}$ if $S$ is closed. One may control the size of the triangles too (see [7] or [5]).

To get a triangulation which is close to $S$ (for the Hausdorff distance), we work as follows (in the particular case where $S$ has no boundary, otherwise it is a little more complicated): We consider a (good) $\tilde{\varepsilon}$-sample (with $\tilde{\varepsilon}<0.1$ ). We fix $\varepsilon>0$. To each face $\Delta$ of $\operatorname{Del}(\mathcal{S})$, we associate the (unique) sphere whose centre $c$ is on $S$ and which contains the vertices of $\sigma$ (we denote its radius by $r_{\Delta}$ ). We consider all the faces $\Delta$ of $\operatorname{Del}(\mathcal{S})$ whose radius $r_{\Delta}$ is larger than $\varepsilon$ or for which one angle is too small. We add the corresponding centres $c_{\mathcal{S}}^{\prime}$ to $\mathcal{S}$ and we build $\operatorname{Del}\left(\mathcal{S} \cup\left\{c_{\mathcal{S}}^{\prime}\right\}\right)$ and $\operatorname{Del}_{\mathcal{S}}\left(\mathcal{S} \cup\left\{c_{\mathcal{S}}^{\prime}\right\}\right)$. Iterating this process, we obtain a triangulation $T(\varepsilon)$ such that

- the length of every edge is $<2 \varepsilon$,

- the fatness is bounded from below by a universal constant (independent of $\varepsilon$ ).

This algorithm terminates (see [7] or [5]). Furthermore, if $\varepsilon<0.08$ and if $S$ has no boundary, then $T(\varepsilon)$ is closely inscribed in $S$ [3]. In that case it is easy to see that the Hausdorff distance between $S$ and the final triangulation is less than $\varepsilon$.

In particular, using Corollary 1 we get the following:

Corollary 10. Let $S$ be a smooth (compact without boundary) surface and let $\mathcal{S}$ be a (good) $\varepsilon$-sample of $S$. Let $\varepsilon>0$. Then the triangulation $T(\varepsilon)$ obtained by the previous algorithm is such that the angle $\alpha_{\max }$ between the normals of $S$ and $T(\varepsilon)$ satisfies

$$
\sin \alpha_{\max } \leq C\left(\rho_{S}\right) \varepsilon,
$$

where $C\left(\rho_{S}\right)$ denotes a constant depending only on the maximal curvature $\rho_{S}$ of $S$.

Consider now the sequence $\varepsilon_{n}=1 / n$. By the previous process, we associate a triangulation $T_{n}$ satisfying the following properties:

- each $T_{n}$ is closely inscribed in $S$;

- the Hausdorff distance between $T_{n}$ and $S$ tends to zero when $n$ tends to infinity;

- the fatness of $T_{n}$ is uniformly bounded from below by a positive constant.

This implies, in particular (by using Corollary 7),

$$
\lim _{n \rightarrow \infty} \mathcal{A}\left(T_{n}\right)=\mathcal{A}(S)
$$


Moreover, we can apply classical results on geometric measure theory, and deduce immediately that the curvature measures of $T_{n}$ converge to the curvature measures of $S$ for the flat topology, and in particular for the weak topology (see [9] and [10] for the background and details on curvature measures).

\section{Proof of Theorem 1}

We first need some technical lemmas.

\subsection{A Purely Geometric Result}

The following lemma is a purely geometric result in $E^{3}$ :

Lemma 1. Let $\Delta$ be a triangle whose vertices are $p, p_{1}$ and $p_{2}$. If $\alpha_{p} \in[0, \pi / 2]$ denotes the angle between a normal to the triangle and the axis $(O, z)$, then

1. $\cos ^{2}\left(\alpha_{p}\right)=\frac{\cos ^{2} \theta_{1} \cos ^{2} \theta_{2}-\sin ^{2} \theta_{1} \sin ^{2} \theta_{2}-\cos ^{2} \gamma+2 \cos \gamma \sin \theta_{1} \sin \theta_{2}}{\sin ^{2}\left(\theta_{2}-\theta_{1}\right)+2 \sin \theta_{1} \sin \theta_{2} \cos \left(\theta_{2}-\theta_{1}\right)+\cos ^{2} \theta_{1} \cos ^{2} \theta_{2}-\sin ^{2} \theta_{1} \sin ^{2} \theta_{2}-\cos ^{2} \gamma}$, where $\theta_{i} \in[-\pi / 2, \pi / 2]$ is the angle between $\overrightarrow{p p}_{i}$ and the orthogonal projection of $\overrightarrow{p p}_{i}$ onto the plane orthogonal to $(O, z)$ which contains $p\left(\theta_{i} \geq 0\right.$ iff the third component of $\overrightarrow{p p}_{i}$ is positive) and $\left.\gamma \in\right] 0, \pi[$ is the angle of $\Delta$ at $p$.

2. In particular, if $\left|\sin \theta_{1}\right| \leq \varepsilon$ and $\left|\sin \theta_{2}\right| \leq \varepsilon$, then

$$
\sin \left(\alpha_{p}\right) \leq \frac{\sqrt{10} \varepsilon}{\sin \gamma}
$$

where $\gamma \in] 0, \pi[$ is the angle of $\Delta$ at $p$.

Proof. 1. We put

$$
\eta_{1}=p p_{1}, \quad \eta_{2}=p p_{2} \quad \text { and } \quad N=\left(\begin{array}{l}
0 \\
0 \\
1
\end{array}\right) .
$$

We can assume that $p=0$. In spherical coordinates, we get

$$
\begin{gathered}
p=\left(\begin{array}{l}
0 \\
0 \\
0
\end{array}\right), \quad p_{1}=\left(\begin{array}{c}
\eta_{1} \cos \varphi_{1} \cos \theta_{1} \\
\eta_{1} \sin \varphi_{1} \cos \theta_{1} \\
\eta_{1} \sin \theta_{1}
\end{array}\right), \quad p_{2}=\left(\begin{array}{c}
\eta_{2} \cos \varphi_{2} \cos \theta_{2} \\
\eta_{2} \sin \varphi_{2} \cos \theta_{2} \\
\eta_{2} \sin \theta_{2}
\end{array}\right), \\
\text { with }\left\{\begin{array}{l}
\theta_{i} \in\left[-\frac{\pi}{2}, \frac{\pi}{2}\right], \\
\varphi_{i} \in[0,2 \pi] .
\end{array}\right.
\end{gathered}
$$


We put

$$
\overrightarrow{p p}_{1} \wedge \overrightarrow{p p}_{2}=\left(\begin{array}{l}
a \\
b \\
c
\end{array}\right)
$$

Thus

$$
\cos \left(\alpha_{p}\right)=\left|\left\langle N, \frac{\overrightarrow{p \vec{p}_{1} \wedge \vec{p}_{2}}}{\left\|\vec{p}_{1} \wedge \vec{p}_{2}\right\|}\right\rangle\right|=\sqrt{\frac{c^{2}}{a^{2}+b^{2}+c^{2}}}
$$

We have

$$
\begin{aligned}
a & =\eta_{1} \eta_{2}\left(\cos \theta_{1} \sin \varphi_{1} \sin \theta_{2}-\cos \theta_{2} \sin \varphi_{2} \sin \theta_{1}\right), \\
b & =\eta_{1} \eta_{2}\left(\cos \theta_{2} \cos \varphi_{2} \sin \theta_{1}-\cos \theta_{1} \cos \varphi_{1} \sin \theta_{2}\right), \\
c & =\eta_{1} \eta_{2} \cos \theta_{1} \cos \theta_{2}\left(\cos \varphi_{1} \sin \varphi_{2}-\sin \varphi_{1} \cos \varphi_{2}\right) \\
& =\eta_{1} \eta_{2} \cos \theta_{1} \cos \theta_{2} \sin \left(\varphi_{2}-\varphi_{1}\right) .
\end{aligned}
$$

This implies that

$$
\begin{aligned}
\frac{a^{2}+b^{2}}{\eta_{1}^{2} \eta_{2}^{2}}= & \cos ^{2} \theta_{1} \sin ^{2} \theta_{2}+\cos ^{2} \theta_{2} \sin ^{2} \theta_{1} \\
& -2 \cos \theta_{1} \cos \theta_{2} \sin \theta_{1} \sin \theta_{2} \cos \left(\varphi_{2}-\varphi_{1}\right) \\
= & \sin ^{2}\left(\theta_{2}-\theta_{1}\right)+2 \cos \theta_{1} \cos \theta_{2} \sin \theta_{1} \sin \theta_{2}\left(1-\cos \left(\varphi_{2}-\varphi_{1}\right)\right) .
\end{aligned}
$$

On the other hand, we get

$$
\begin{aligned}
\cos \gamma & =\frac{\left\langle\overrightarrow{p p}_{1}, \overrightarrow{p p_{2}}\right\rangle}{\eta_{1} \eta_{2}} \\
& =\cos \theta_{1} \cos \theta_{2}\left(\cos \varphi_{1} \cos \varphi_{2}+\sin \varphi_{1} \sin \varphi_{2}\right)+\sin \theta_{1} \sin \theta_{2} \\
& =\cos \theta_{1} \cos \theta_{2} \cos \left(\varphi_{2}-\varphi_{1}\right)+\sin \theta_{1} \sin \theta_{2} .
\end{aligned}
$$

Suppose that $\cos \theta_{1} \neq 0$ and $\cos \theta_{2} \neq 0$. Then

$$
\cos \left(\varphi_{2}-\varphi_{1}\right)=\frac{\cos \gamma-\sin \theta_{1} \sin \theta_{2}}{\cos \theta_{1} \cos \theta_{2}} .
$$

Therefore

$$
\begin{aligned}
\frac{a^{2}+b^{2}}{\eta_{1}^{2} \eta_{2}^{2}}= & \sin ^{2}\left(\theta_{2}-\theta_{1}\right)+2 \cos \theta_{1} \cos \theta_{2} \sin \theta_{1} \sin \theta_{2} \\
& \times\left(1-\frac{\cos \gamma-\sin \theta_{1} \sin \theta_{2}}{\cos \theta_{1} \cos \theta_{2}}\right) \\
= & \sin ^{2}\left(\theta_{2}-\theta_{1}\right)+2 \sin \theta_{1} \sin \theta_{2} \cos \left(\theta_{2}-\theta_{1}\right)-2 \cos \gamma \sin \theta_{1} \sin \theta_{2},
\end{aligned}
$$




$$
\begin{aligned}
\frac{c^{2}}{\eta_{1}^{2} \eta_{2}^{2}}= & \cos ^{2} \theta_{1} \cos ^{2} \theta_{2}\left(1-\left(\frac{\cos \gamma-\sin \theta_{1} \sin \theta_{2}}{\cos \theta_{1} \cos \theta_{2}}\right)^{2}\right) \\
= & \cos ^{2} \theta_{1} \cos ^{2} \theta_{2}-\sin ^{2} \theta_{1} \sin ^{2} \theta_{2}-\cos ^{2} \gamma+2 \cos \gamma \sin \theta_{1} \sin \theta_{2}, \\
\frac{a^{2}+b^{2}+c^{2}}{\eta_{1}^{2} \eta_{2}^{2}}= & \sin ^{2}\left(\theta_{2}-\theta_{1}\right)+2 \sin \theta_{1} \sin \theta_{2} \cos \left(\theta_{2}-\theta_{1}\right) \\
& +\cos ^{2} \theta_{1} \cos ^{2} \theta_{2}-\sin ^{2} \theta_{1} \sin ^{2} \theta_{2}-\cos ^{2} \gamma .
\end{aligned}
$$

Suppose now that $\cos \theta_{1}=0$. Thus the angle $\alpha_{p}$ is equal to $\pi / 2$ or $-\pi / 2$ and $\cos ^{2} \alpha_{p}=0$. Since $\cos \gamma=\sin \theta_{2}$, the result is still true.

2. We have

$$
\left|\sin \theta_{i}\right| \leq \varepsilon \quad \text { and } \quad\left|\cos \theta_{i}\right| \geq \sqrt{1-\varepsilon^{2}}
$$

Thus

$$
\begin{aligned}
\cos ^{2} \theta_{1} \cos ^{2} \theta_{2}-\sin ^{2} \theta_{1} \sin ^{2} \theta_{2}-\cos ^{2} \gamma+2 \cos \gamma \sin \theta_{1} \sin \theta_{2} \\
\quad \geq\left(1-\varepsilon^{2}\right)-\varepsilon^{4}-\cos ^{2} \gamma-2 \varepsilon^{2} \\
\quad=\sin ^{2} \gamma-4 \varepsilon^{2}
\end{aligned}
$$

and

$$
\begin{aligned}
\sin ^{2}\left(\theta_{2}-\right. & \left.\theta_{1}\right)+2 \sin \theta_{1} \sin \theta_{2} \cos \left(\theta_{2}-\theta_{1}\right)+\cos ^{2} \theta_{1} \cos ^{2} \theta_{2}-\sin ^{2} \theta_{1} \sin ^{2} \theta_{2}-\cos ^{2} \gamma \\
& \leq 4 \varepsilon^{2}+2 \varepsilon^{2}+1-\cos ^{2} \gamma \\
& =\sin ^{2} \gamma+6 \varepsilon^{2} .
\end{aligned}
$$

Then

$$
\cos ^{2}\left(\alpha_{p}\right) \geq \frac{\sin ^{2} \gamma-4 \varepsilon^{2}}{\sin ^{2} \gamma+6 \varepsilon^{2}}
$$

Suppose now that $6 \varepsilon^{2} / \sin ^{2} \gamma<1$. Then we get

$$
\cos ^{2}\left(\alpha_{p}\right) \geq\left(1-\frac{4 \varepsilon^{2}}{\sin ^{2} \gamma}\right)\left(1-\frac{6 \varepsilon^{2}}{\sin ^{2} \gamma}\right) \geq 1-\frac{10 \varepsilon^{2}}{\sin ^{2} \gamma}
$$

and

$$
\sin \left(\alpha_{p}\right) \leq \frac{\sqrt{10} \varepsilon}{\sin \gamma}
$$

If $6 \varepsilon^{2} / \sin ^{2} \gamma \geq 1$, the result is still true.

\subsection{Comparing the Length of a Geodesic and Its Chord}

We need the following result that gives the main differential properties of the function $\xi$ when it is defined. It has been studied in [11] in the case where $S$ is the boundary of a convex set of $E^{3}$ and in [14]. 
Proposition 3. Let $S$ be a smooth surface of $E^{3}$ without boundary, let $U_{S}$ be an open subset of $E^{3}$ where the map $\xi: U_{S} \rightarrow S$ is well defined. Then:

1. The map $\xi$ is $C^{1}$ in $U_{S}$ and satisfies, for every $m \in U_{S}$,

$$
\begin{aligned}
& D \xi(m)\left(Z_{m}\right)=0, \quad \forall Z_{m} \text { orthogonal to } T_{\xi(m)} S, \\
& D \xi(m)\left(X_{m}\right)=\left(I d+\delta_{m} \varepsilon_{m} A_{\xi(m)}\right)^{-1}\left(X_{m}\right), \quad \forall X_{m} \text { parallel to } T_{\xi(m)} S,
\end{aligned}
$$

where $\varepsilon_{m}=\left\langle v_{\xi(m)},(\xi(m)-m) /\|\xi(m)-m\|\right\rangle \in\{-1,+1\}$ and $A_{\xi(m)}=-D v$ $(\xi(m))$ is the Weingarten endomorphism of $S$ at the point $\xi(m)$.

2. In particular, the matrix of $D \xi(m): E^{3} \rightarrow T_{\xi(m)} S$ (in local orthonormal frames $\left(e_{\xi(m)}^{1}, e_{\xi(m)}^{2}, v_{\xi(m)}^{S}\right)$ and $\left.\left(e_{\xi(m)}^{1}, e_{\xi(m)}^{2}\right)\right)$ is given by

$$
\left(\begin{array}{ccc}
\frac{1}{1+\delta_{m} \varepsilon_{m} \lambda_{\xi(m)}^{1}} & 0 & 0 \\
0 & \frac{1}{1+\delta_{m} \varepsilon_{m} \lambda_{\xi(m)}^{2}} & 0
\end{array}\right)
$$

where $e_{\xi(m)}^{1}$ and $e_{\xi(m)}^{2}$ are unitary principal vectors and $v_{\xi(m)}^{S}$ is the oriented normal of $S$ at $\xi(m)$.

Sketch of proof.

- For every $m \in U_{S}$, the point $\xi(m)$ is defined by the following relation:

$$
\forall m \in U_{S}, \quad \forall X \in T_{\xi(m)} S, \quad\left\langle\xi(m)-m, X_{\xi(m)}\right\rangle=0 .
$$

Consequently, for every $m \in T_{m} S$, the function $\xi$ is constant on the orthogonal of $T_{m} S$.

- Consider now a vector $X_{m} \in T_{m} U_{S}$ which is parallel to $T_{\xi(m)} S$. We have

$$
D \xi(m)(X)=X+\delta_{m} \varepsilon_{m} D v(\xi(m)) \circ D \xi(m)(X) .
$$

The endomorphism $\left(I+\delta_{m} \varepsilon_{m} A_{\xi(m)}\right)$ is clearly invertible, and, consequenly,

$$
D \xi(m)(X)=\left(I+\delta_{m} \varepsilon_{m} A_{\xi(m)}\right)^{-1}(X) .
$$

- The rest of the proof is obvious.

Corollary 11. Let $S$ be a smooth compact surface of $E^{3}$, let $U_{S}$ be a neighbourhood of $S$ where the map $\xi: U_{S} \rightarrow S$ is well defined, and let $p$ and $q$ be two points on $S$ such that $[p, q] \subset U_{S}$ and $\xi(] p, q[) \subset S \backslash \partial S$. Then the distance $l_{p q}$ between $p$ and $q$ on $S$ satisfies

$$
l_{p q} \leq \frac{1}{1-\omega} p q,
$$

where $\omega=\sup _{m \in] p, q[}\|\xi(m)-m\| \rho_{\xi(m)}$. 
Proof. Since $\xi([p, q])$ is a curve on $S$, its length is larger than the length $l_{p q}$ of the geodesic whose ends are $p$ and $q$. Therefore,

$$
l_{p q} \leq l(\xi([p, q])) \leq \sup _{m \in] p, q[}|D \xi(m)| p q .
$$

On the other hand, for every $m \in] p, q[$, we have (see the remarks above)

$$
\|\xi(m)-m\| \rho_{\xi(m)}<1 .
$$

Since $\xi(p)=p$ and $\xi(q)=q, \omega<1$. Therefore Proposition 3 implies that

$$
|D \xi(m)| \leq \frac{1}{1-\|\xi(m)-m\| \rho_{\xi(m)}} \leq \frac{1}{1-\omega},
$$

and Corollary 11 is proved.

\subsection{Comparing the Normals at a Vertex}

We shall prove the following:

Proposition 4. Let $S$ be a smooth surface, let $\Delta$ be a triangle closely inscribed in $S$ and let $p$ be a vertex of $\Delta$. Then the angle $\alpha_{p} \in[0, \pi / 2]$ between the normals of $S$ and $\Delta$ at $p$ satisfies

$$
\sin \left(\alpha_{p}\right) \leq \frac{\sqrt{10} \pi_{S}(\Delta)}{2 \sin \gamma_{p}\left(1-\omega_{S}(\Delta)\right)},
$$

where $\gamma_{p}$ is the angle of $\Delta$ at $p$.

This proposition is a consequence of the following:

Lemma 2. Let $S$ be a smooth surface and let $p, q \in S$ such that $[p, q] \subset S$. Then the angle $\theta \in[0, \pi / 2]$ between $\overrightarrow{p q}$ and the orthogonal projection of $\overrightarrow{p q}$ onto $T_{p} S$ satisfies

$$
\sin \theta \leq \frac{\rho_{S} l}{2}
$$

where $l$ is the distance on $S$ between $p$ and $q$.

Proof. Let $\mathcal{C}$ denote a geodesic of $S$ linking $p$ and $q . \mathcal{C}$ is parametrized by arc length by

$$
\gamma:[0, l] \rightarrow S,
$$

with $\gamma(0)=p$ and $\gamma(l)=q$. A simple calculation gives

$$
\gamma(l)-\gamma(0)=l \gamma^{\prime}(0)+\int_{0}^{l}(l-t) \gamma^{\prime \prime}(t) d t,
$$


thus

$$
\gamma^{\prime}(0)=\frac{\overrightarrow{p q}}{l}-\frac{1}{l} \int_{0}^{l}(l-t) \gamma^{\prime \prime}(t) d t .
$$

Let $\vec{v}=(1 / l) \int_{0}^{l}(l-t) \gamma^{\prime \prime}(t) d t$ and $\vec{e}=\overrightarrow{p q} / p q$. We have

$$
\gamma^{\prime}(0)=\frac{p q}{l} \vec{e}-\vec{v} \quad \text { with } \quad\left\{\begin{array}{l}
\|\vec{e}\|=1, \\
\|\vec{v}\| \leq \frac{l \rho_{\xi_{s}}}{2},
\end{array}\right.
$$

and

$$
\sin \theta=\inf _{\substack{\vec{u} \in T_{p} S \\\|\vec{u}\|=1}}\|\vec{u} \wedge \vec{e}\| \leq \frac{l \rho_{S}}{2} .
$$

Proof of Proposition 4. Denote by $l_{1}$ the distance on $S$ between $p$ and $p_{1}$, and by $l_{2}$ the distance on $S$ between $p$ and $p_{2}$. Since $T$ is closely inscribed in $S$, thanks to Corollary 11 we get

$$
l_{1} \leq \frac{p p_{1}}{1-\omega_{S}(\Delta)} \leq \frac{\eta_{\Delta}}{1-\omega_{\Delta}} \quad \text { and } \quad l_{2} \leq \frac{\eta_{\Delta}}{1-\omega_{\Delta}} .
$$

Therefore, Lemma 2 implies that

$$
\sin \theta_{1} \leq \frac{\rho_{\xi(\Delta)} l_{1}}{2} \leq \frac{\pi_{S}(\Delta)}{2\left(1-\omega_{S}(\Delta)\right)} \quad \text { and } \quad \sin \theta_{2} \leq \frac{\pi_{S}(\Delta)}{2\left(1-\omega_{S}(\Delta)\right)} .
$$

Then Lemma 1 implies

$$
\sin \left(\alpha_{p}\right) \leq \frac{\sqrt{10}}{\sin \gamma_{p}} \frac{\pi_{S}(\Delta)}{2\left(1-\omega_{S}(\Delta)\right)}=\frac{\sqrt{10} \pi_{S}(\Delta)}{2 \sin \gamma_{p}\left(1-\omega_{S}(\Delta)\right)} .
$$

\subsection{Comparing the Normals of a Smooth Surface}

Proposition 5. Let $S$ be a smooth compact oriented surface of $E^{3}$, let $\Delta$ be a triangle closely inscribed in $S$ and let $p$ and $s$ be two points on $\Delta$. Then the angle $\alpha_{s p} \in[0, \pi / 2]$ between two normals $v_{\xi(p)}^{S}$ and $v_{\xi(s)}^{S}$ at $\xi(p)$ and $\xi(s)$ satisfies

$$
\sin \left(\alpha_{s p}\right) \leq \frac{\pi_{S}(\Delta)}{1-\omega_{\Delta}}
$$

where $\eta_{\Delta}$ is the height of $\Delta$ and $\omega_{\Delta}$ is the relative curvature of $\Delta$ with respect to $\xi(\Delta)$.

This proposition is the consequence of the following lemma, which is a direct application of the mean value theorem:

Lemma 3. Let $S$ be a smooth compact oriented surface of $E^{3}$ and let $a$ and $b$ be two points of $S$. The angle $\alpha_{a b} \in[0, \pi / 2]$ between two normals $v_{a}^{S}$ and $v_{b}^{S}$ at $a$ and $b$ satisfies

$$
\sin \left(\alpha_{a b}\right) \leq \rho_{S} L_{S}(a, b),
$$


where $\rho_{S}$ is the maximal curvature of $S$ and $L_{S}(a, b)$ is the distance on $S$ between a and $b$.

The proof of Proposition 5 is an obvious consequence of Lemma 3 and Proposition 3.

Theorem 1 can be immediately deduced from Propositions 4 and 5, since

$$
\sin \left(\alpha_{s}\right) \leq \sin \left(\alpha_{p}\right)+\sin \left(\alpha_{s p}\right) .
$$

\section{Proof of Theorem 2}

Consider the surface $M$ lying in $U_{S}$. Since $M$ is compact and locally lipschitz, there exists a finite family of lipschitz graphs $M_{i}$, such that $M=\bigcup_{i} M_{i}$ and $M_{i} \cap M_{j}=\emptyset$ (if $i \neq j)$. We put $S_{i}=\xi\left(M_{i}\right)$. By assumption, the restriction $\xi_{\mid M_{i}}$ of $\xi$ to $M_{i}$ is one-to-one. Furthermore, the function $\xi$ is differentiable at every point of $M$. We apply the general area-coarea formula [13]:

$$
\mathcal{A}\left(S_{i}\right)=\int_{S_{i}} d a_{S_{i}}(s)=\int_{M_{i}}\left|J a c_{2} D \xi(m)\right| d a_{M_{i}}(m),
$$

where $J a c_{2} D \xi(m)$ is the two-dimensional Jacobian of $\xi$ at $m$. Since $M_{i}$ is a lipschitz surface, $M_{i}$ admits a tangent plane at almost every point $m$. In such a point $m, J a c_{2} D \xi(m)$ equals the determinant of the linear map

$$
D \xi_{\mid M_{i}}: T_{m} M_{i} \rightarrow T_{\xi(m)} S .
$$

We have

$$
\mathcal{A}\left(S_{i}\right)=\int_{M_{i}}\left|D \xi_{\mid M_{i}}(m)\right| d a_{M_{i}}(m)=\int_{M_{i}}\left|D \xi_{\mid M}(m)\right| d a_{M}(m) .
$$

Therefore, by taking the sum over all the $M_{i}$ 's, we get

$$
\left.\mathcal{A}(S)=\int_{M} \mid D \xi_{\mid M}(m)\right) \mid d a_{M}(m) .
$$

The following lemma evaluates the Jacobian of $\xi_{\mid M}$.

Lemma 4. Let $S$ be a smooth surface of $E^{3}$ without boundary and let $U_{S}$ be an open subset of $E^{3}$ where the map $\xi: U_{S} \rightarrow S$ is well defined. Let $M \subset U_{S}$ be a smooth surface. Then the Jacobian of the differential of $\xi_{\mid M}$ is given by

$$
\left|D \xi_{\mid M}(m)\right|=\frac{\cos \alpha_{m}}{\left(1+\delta_{m} \varepsilon_{m} \lambda_{\xi(m)}^{1}\right)\left(1+\delta_{m} \varepsilon_{m} \lambda_{\xi(m)}^{2}\right)},
$$

where $\varepsilon_{m}=\left\langle\nu_{\xi(m)},(\xi(m)-m) /\|\xi(m)-m\|\right\rangle \in\{-1,+1\}$, and $\delta_{m}=\|\xi(m)-m\|$.

This lemma is obvious, by considering the restriction $\xi_{\mid M}$ of $\xi$ to $M$. Note that the condition of the theorem on the angle between corresponding tangent planes implies that $\xi_{\mid M}$ is a diffeomorphism between $M$ and $S$. 
End of the Proof of Theorem 2. Since $M$ is closely inscribed in $S, \xi_{\mid M}$ is one-to-one between $M$ and $S$. Therefore,

$$
\mathcal{A}(S)=\int_{M}\left|D \xi_{\mid M}(m)\right| d a_{M}(m)=\int_{M} \frac{\cos \alpha_{m}}{\left(1+\delta_{m} \varepsilon_{m} \lambda_{\xi(m)}^{1}\right)\left(1+\delta_{m} \varepsilon_{m} \lambda_{\xi(m)}^{2}\right)} d a_{M}(m)
$$

\section{Conclusion}

In this paper we give a very general result relating the normal vector field of a smooth surface and the normal vector field of a triangulation. This result induces a result of the approximation of the area of a smooth surface with the area of a triangulation. These results are applied in surface reconstruction. We hope that this kind of result can be useful to get a general theoretical approach to the approximation of the geometrical properties of a smooth surface and a triangulation close to it.

\section{References}

1. N. Amenta, M. Bern, Surface reconstruction by Voronoi filtering, Discrete Comput. Geom. 22 (1999), 481-504.

2. N. Amenta, M. Bern, M. Kamvysselis, A new Voronoi-based surface reconstruction algorithm, Proc. Siggraph'98 (1998), pp. 415-421.

3. N. Amenta, S. Choi, T.K. Dey, N. Leekha, A simple algorithm for homeomorphic surface reconstruction, Proc. 16th Ann. Symp. on Computational Geometry (2000), pp. 213-222.

4. M. Berger, B. Gostiaux, Géométrie différentielle: variétés, courbes et surfaces, seconde édition, Presses Universitaires de France, Paris, 1992.

5. J.D. Boissonnat, Diagrammes de Voronoï, Triangulations et surfaces, cours de DEA, novembre 2001.

6. J.D. Boissonnat, F. Cazals, Smooth surface reconstruction via natural neighbor interpolation of distance functions, In Proc. 16th Ann. Symp. on Computational Geometry (2000), pp. 223-232.

7. L.P. Chew, Guaranteed-quality mesh generation for curved surfaces, Proc. 9th Ann. Computational Geometry (1993), pp. 274-280.

8. M. P. Do Carmo, Differential Geometry of Curves and Surfaces (translated from the Portuguese), PrenticeHall, Englewood Cliffs, NJ, 1976.

9. H. Federer, Curvature measures, Trans. Amer. Math. Soc. 93 (1959), 418-491.

10. J. Fu, Convergence of curvatures in secant approximations, J. Differential Geom. 37 (1993), 177-190.

11. A. Haraux, How to differentiate the projection on a convex set in Hilbert space. Some applications to variational inequalities, J. Math. Soc. Japan 29(4) (1977), 615-631.

12. J. Milnor, Morse Theory, Princeton University Press, Princeton, NJ, 1963.

13. F. Morgan, Geometric Measure Theory, Academic Press, New York, 1987.

14. J.M. Morvan, B. Thibert, On the approximation of a smooth surface with a triangulated mesh, Comput. Geom. Theory Appl. 23(3) (2002), 337-352.

15. M. Spivak, A Comprehensive Introduction to Differential Geometry, Vol. III, second edition, Publish or Perish, Wilmington, DE, 1979. 